\title{
THE HIGH-DENSITY MULTIWIRE DRIFT CHAMBER
}

\author{
A. P. JEAVONS, G. CHARPAK and R. J. STUBBS \\ Cern, Geneva, Switzerland
}

Received 12 December 1974

A multiwire proportional chamber, with a high-density drift space attached, has been developed as a position-sensitive detector for non-ionizing radiation.

A chamber with a $5 \mathrm{~g} / \mathrm{cm}^{3}$ drift space, comprising a leadbismuth matrix of $1 \mathrm{~mm}$ square holes on a $1.5 \mathrm{~mm}$ pitch has been investigated. For $0.66 \mathrm{MeV}$ photons a detection efficiency of $5 \%$,

\section{Introduction}

Experimental work involving the spatial localization of ionizing particles has advanced in recent years following the development of the multiwire proportional chamber $(\mathrm{MPC})^{1}$ ). Large systems involving many thousands of wires are now in operation. Progress in this field continues with the drift chamber ${ }^{1-3}$ ), which offers the possibility of submillimetre resolution and very large $(4 \mathrm{~m} \times 4 \mathrm{~m})$, comparatively cheap, detectors.

Increasing attention is now being paid to the use of MPCs as position-sensitive detectors for non-ionizing radiation. This is hardly surprising in view of the many possible applications. The uses of MPCs for medical $X$-ray imaging ${ }^{4}$ ), X-ray crystallography ${ }^{5}$ ) and neutron detection $^{6}$ ) have been reported; other fields of application include $\mathrm{X}$ - and gamma-ray astronomy, solid-state physics, and non-destructive material testing.

The detection of non-ionizing radiation presents two problems for the MPC:

i) The conversion of the non-ionizing radiation into ionizing particles.

ii) Obtaining $X$ and $Y$ information from one chamber, since, in general, the ionizing products will either not propagate to a second chamber or else will be strongly scattered before reaching it.

The second problem is relatively easily resolved. Various two-dimensional read-out techniques: pulserise-time ${ }^{7}$ ), delay-line ${ }^{8}$ ), charge-division ${ }^{9}$ ) and centreof-gravity ${ }^{10}$ ) have been described, offering different combinations of cost, accuracy, sensitivity, and data rate.

To date, the first problem has received little detailed attention. Effort has centred on using the gas in the $\mathrm{MPC}$ as the converting material. For $8 \mathrm{keV} \mathrm{X}$ rays, gas conversion is very successful. $100 \%$ efficiency combined combined with a spatial resolution of $1.3 \mathrm{~mm}$ fwhm, is obtained. Much higher efficiencies are possible. A theoretical analysis of the detection efficiency as a function of photon energy is presented. It agrees well with experimental results.

The chamber offers new possibilities for photon and neutron detection, and as a shower detector for high-energy particles.

with submillimetre spatial resolution is possible using argon at atmospheric pressure. Recently, the introduction of the spherical multiwire drift chamber ${ }^{11}$ ) has extended this efficiency and spatial resolution to the cases of X-ray imaging from a point source or from a divergent beam.

As photon energy is increased, gas conversion becomes less and less useful. Firstly, the photoelectric capture cross section falls very rapidly and, secondly, the range of the photoelectron increases. Thus efficiency and spatial resolution both degrade. By using a dense gas with a high atomic number, under pressure, the photon energy at which the chamber performs satisfactorily can be maximized. Xenon at $4 \mathrm{~atm}$ pressure has been used for $60 \mathrm{keV}$ gamma rays to obtain an efficiency of $50 \%$ and a spatial resolution of $1 \mathrm{~mm}^{4}$ ). This would seem to be a useful limit for gaseous converters.

Carrying the idea of pressure to its logical conclusion leads to a chamber filled with a liquid. And indeed, liquid-argon- and liquid-xenon-filled chambers have been investigated. For $0.28 \mathrm{MeV}$ gamma rays an efficiency of $65 \%$ and a resolution of $4 \mathrm{~mm}$ has been recorded ${ }^{12}$ ). However, serious technical problems exist due to the low-temperature operation, the very high liquid purity needed, and the fact that avalanche amplification is difficult to obtain. The chambers must operate in the ionization region with consequent electronic problems and cost.

As an alternative to a liquid-filled chamber, an ordinary gas-filled chamber may be equipped with a solid converter. Then the advantages of the solid - a high capture efficiency for gamma rays and short range of the resulting photo-electron - may be obtained, together with the normal operation of the gas-filled MPC. Such a hybrid chamber and its application to 
photon energies greater than $0.1 \mathrm{MeV}$, i.e. above the gas-conversion limit, is the subject of this paper.

\section{A gas-solid hybrid chamber}

\subsection{THE BASIC DESIGN}

A dilemma arises for the detection of the ionizing products resulting from the conversion of non-ionizing radiation. If sufficient bulk material is used to give a high capture probability for the non-ionizing radiation, then generally the ionizing products will have a low probability of escaping from the material and being detected. This dilemma is neatly overcome with the noble gases. The free electrons resulting from ionization may be transported, or drifted, over a distance of many centimetres under the influence of an electric field, and then detected. Electron drifting is also possible in liquid argon and xenon. Thus chambers employing a noble gas or liquid as the converting medium can give high detection efficiencies.

The simplest way of using a solid for conversion is to utilize the walls of a chamber. This is how $\mathrm{X}$ and gamma rays are detected with a conventional Geiger counter. With walls made of lead, an efficiency of around $1 \%$ is obtained, over the energy range 0.1 to $2.0 \mathrm{MeV}^{13}$ ).

To obtain a high detection efficiency with a solid, a structure is required where the solid is in thin sections, interleaved with the gas. The photoelectron will then have a high probability of escaping to the gas and creating free electrons which may be extracted with a drift field. To obtain a good spatial resolution, the range of the photoelectron must be restricted to small pockets of gas. A structure that fits these two require-

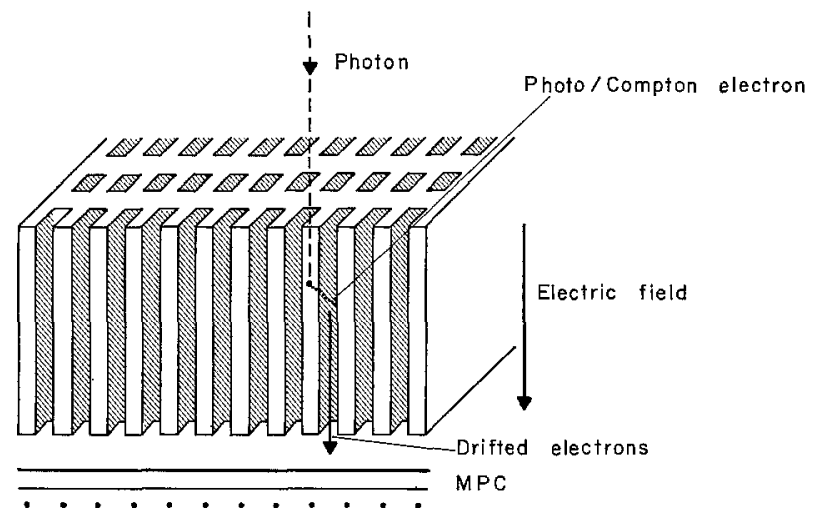

Fig. 1. Principle of the gas-solid hybrid chamber. Photons are captured in the solid bars and produce fast electrons which can escape to an adjacent hole. The free electrons resulting from gas ionization in the hole may be extracted by an electric drift field, and detected by a proportional chamber. ments is a solid block, perforated with a large number of small holes close together. By making the block thick enough, the photon will have a high conversion
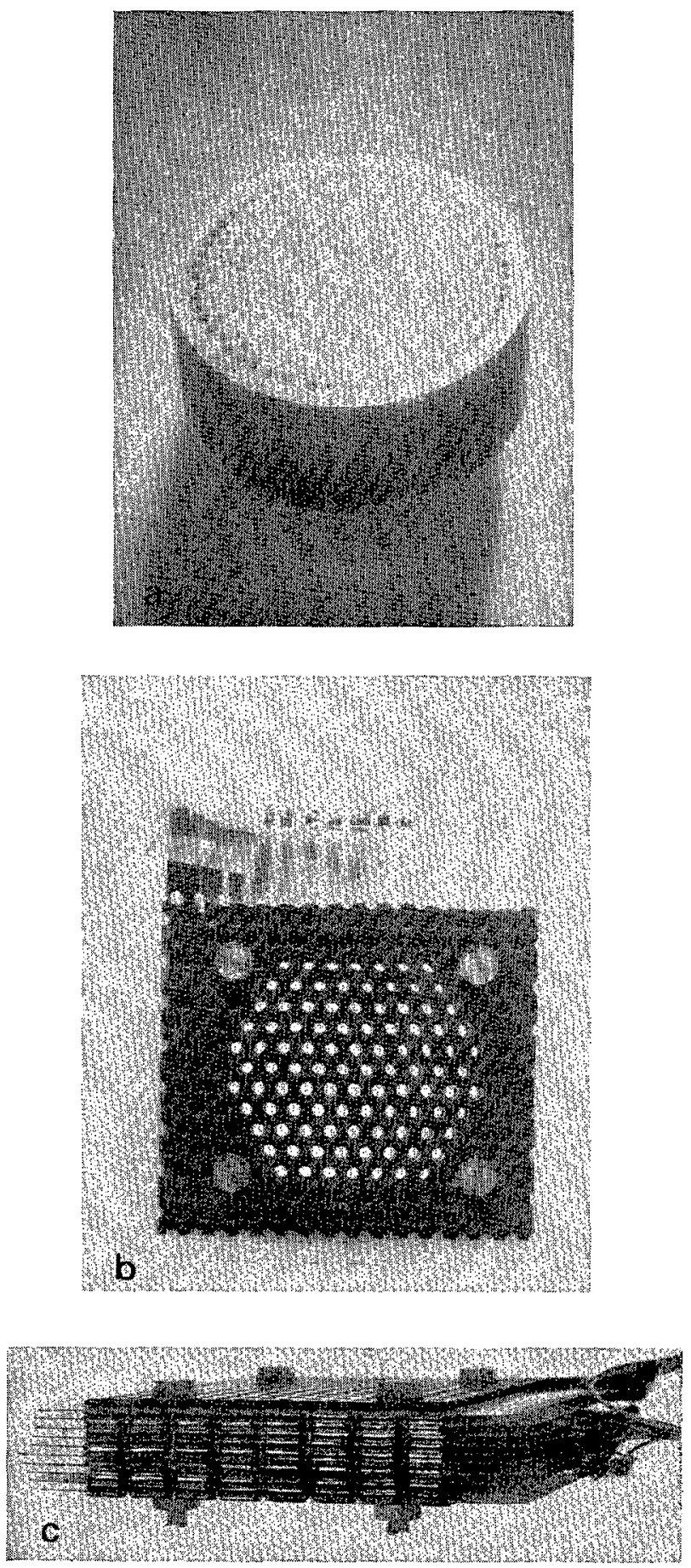

Fig. 2. Different ways of making the solid matrix: (a) a bundle of glass tubes (full size); (b), (c) a copper-plate, mylar sandwich $(\times 2 / 3)$. 
probability. With a correct choice of hole size and spacing, the resulting photoelectron will have a high probability of escaping to a hole but a low probability of propagating to a second hole. The application of an electric field will drift the free electrons out of these holes for subsequent detection by a MPC. If the hole axis is parallel to the direction of the impinging photons, the two dimensions perpendicular to this direction may be resolved to an accuracy determined by the hole size (see fig. 1).

A somewhat similar, independent approach has been reported. An open honeycomb structure was used to provide a large surface area of lead for photons angled at $20^{\circ}$ to the direction perpendicular to the chamber. A detection efficiency of $2.5 \%$, combined with a spatial resolution of $5 \mathrm{~mm}$, was obtained ${ }^{14}$ ).

\subsection{EXPERIMENTAL TESTS}

Two small prototypes were tested to assess different methods of fabricating a solid matrix and to prove that electrons could be drifted efficiently through holes in a solid.
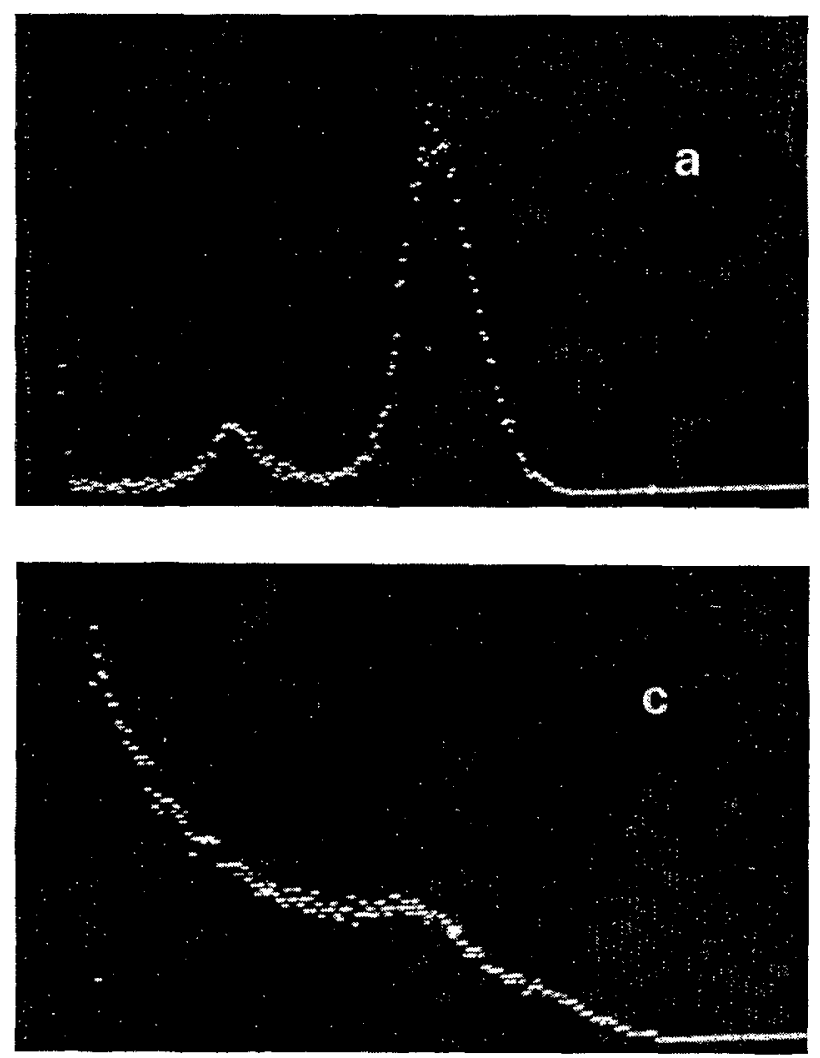

\subsubsection{Glass tubes}

A matrix $4 \mathrm{~cm}$ in diameter and $2 \mathrm{~cm}$ long was made by packing together soda-glass tubes of $1.5 \mathrm{~mm}$ bore and $0.5 \mathrm{~mm}$ wall thickness (see fig.2a). It was placed in a drift space attached to a small $(10 \mathrm{~cm} \times 10 \mathrm{~cm})$ MPC. Steel mesh grids immediately above and below the bundle of tubes provided the drift field. A source of $5.9 \mathrm{keV}{ }^{55} \mathrm{Fe} \mathrm{X}$ rays was placed above the matrix and the count rate and pulse spectrum of the chamber monitored to observe changes with and without the drift voltage applied. Initially the tubes exhibited an electrostatic charging effect. An increase in count rate was observed when the drift voltage was applied, but it decayed rapidly (1-2 min). Following a dipping of the tubes in an antistatic glue, a reasonably stable, eightfold increase in count rate was obtained. Fig. 3 shows the variation in pulse height with drift voltage obtained from one anode wire. With a back-drift voltage applied (fig. 3a) good energy resolution is apparent. This is expected since the tubes will act purely as an X-ray collimator. The application of a forward-drift voltage gave a large increase in the num-
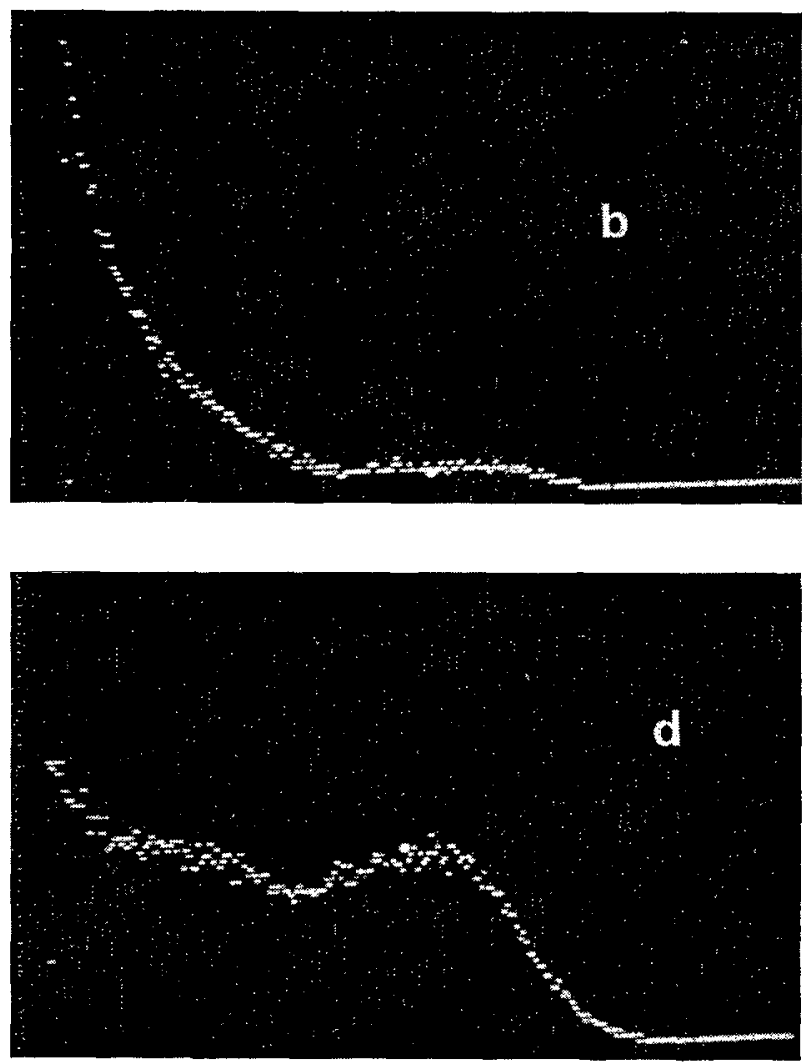

Fig. 3. The variation of pulse-height spectrum with drift voltage for the glass tubes. $5.9 \mathrm{keV}$ photon source. Back-drift voltage. The tubes act solely as an X-ray collimator. (a) $1000 \mathrm{~V}$.

Forward-drift voltage. The count rate increases eightfold. (b) $1000 \mathrm{~V}$; (c) $2000 \mathrm{~V}$; (d) $3000 \mathrm{~V}$. 
ber of low-energy pulses (fig. 3b). Increasing the drift voltage did not further increase the count rate, but flattened the pulse-height spectrum, indicating an increased efficiency of electron collection (see figs. 3c and $3 \mathrm{~d})$.

An important result to observe is the flat spectrum of fig. 3d, which implies an almost complete loss of energy resolution. This is caused by the random amount of energy lost by any photoelectron in interacting with the wall of a glass tube.

Electrostatic charging effects were still seen, particularly if the drift voltage was decreased, when the count rate and pulse-height spectrum would take many minutes to stabilize.

\subsubsection{Metal plates}

To avoid electrostatic charging and to have a denser matrix, a stack of perforated metal plates, insulated from one another, was investigated. Eleven plates of copper $6 \mathrm{~cm} \times 6 \mathrm{~cm} \times 1 \mathrm{~mm}$ were interleaved with mylar sheets $0.1 \mathrm{~mm}$ thick. The complete stack was perforated with $2 \mathrm{~mm}$ diameter holes every $3.5 \mathrm{~mm}$ on a hexagonal pattern (see figs. $2 b$ and $2 c$ ). The drift field within the holes was produced by connecting the plates to a linear resistor chain. Testing with the ${ }^{55} \mathrm{Fe}$ source as before gave similar results, but without any electrostatic charging problems.

In view of the completely stable performance of the metal plates compared to the glass tubes, it was decided to construct a larger chamber of metal plates to make detailed measurements of detection efficiency and spatial resolution. Before this is described, some theoretical questions will be considered.

\section{Theoretical analysis}

The calculation of the detection efficiency of the solid matrix is essentially the problem of calculating the probability that the fast electron produced by a photon interaction will escape from the solid. From first principles this is very difficult, due to the complex nature of the multiple scattering of the fast electron. However, some excellent theoretical [Spencer $\left.{ }^{15,16}\right)$ ] and experimental [Seliger ${ }^{17}$ )] work may be invoked to make the calculation relatively straightforward.

Fig. 4 illustrates a matrix of square holes. The hole size is $h$ and the bar width between holes is $b$. The matrix is made of $n$ plates each of thickness $t$, material density $\rho$ and photon interaction cross section $\sigma$. Consider a line segment along a bar at a distance $x$ from the edge of a hole. The area of this segment is $(h+2 x) \mathrm{d} x$. The unit cell of the matrix has an area $(h+b)^{2}$ and includes 4 such segments, so the area ratio as a function of $x$ is:

$$
\left[4 /(h+b)^{2}\right](h+2 x) \mathrm{d} x \text {. }
$$

Let a photon impinging on the line segment have a probability $P_{\mathrm{e}}(E)$ of producing an electron, energy $E$, which in turn has a probability $P_{\mathrm{d}}(x, E)$ of escaping to a hole and being detected. $P_{\mathrm{d}}(x, E)$ will steadily decrease as $x$ increases. The value of $x$ at which $P_{\mathrm{d}}(x, E)$ goes to zero is the electron residual range, $\left.R(E)^{18}\right)$.

The assumption is made that an electron can only escape to the nearest hole, i.e. $R(E)<b / 2$. Then, if $F$ is the fraction of electrons detected from the photons interacting anywhere in the matrix,

$F=\frac{4}{(h+b)^{2}} \int_{0}^{E_{1}} P_{\mathrm{e}}(E) \int_{0}^{R(E)}(h+2 x) P_{\mathrm{d}}(x, E) \mathrm{d} x \mathrm{~d} E$.

The integration limit $E_{1}$ will be discussed later.

The over-all efficiency, $\varepsilon$, is obtained by including the probability of photon interaction and summing for the relevant processes:

$$
\varepsilon=\left[1-\exp \left(-\sigma_{\mathrm{T}} \rho n t\right)\right] \sum_{i} \frac{\sigma_{i}}{\sigma_{\mathrm{T}}} F_{i},
$$

where $\sigma_{\mathrm{T}}=\underset{i}{\sum_{i}} \sigma_{i}$

\subsection{THE ELECTRON ESCAPE PROBABILITY}

To evaluate $F_{i}$, functions must be found for $P_{\mathrm{e}}(E)$ and $P_{\mathrm{d}}(x, E)$. Spencer $\left.{ }^{15}\right)$ used a numerical method of spatial moments to calculate $P_{\mathrm{d}}(x, E)$, but he also gives an analytic expression:

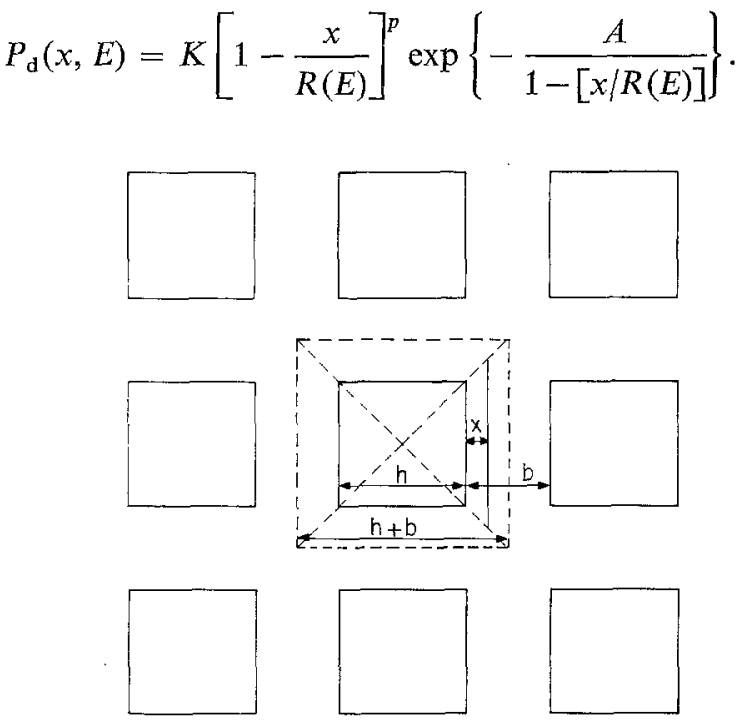

Fig. 4. Parameter details for the theoretical analysis. 
TABLE 1

The values of the coefficient $A$ in eq. (3) obtained from Seliger's data. $A$ is reasonably constant for each material. Note the deviations from Spencer's asymptotic values.

\begin{tabular}{|c|c|c|c|c|c|c|c|c|c|c|c|}
\hline Material & $\begin{array}{l}\text { Energy } \\
(\mathrm{MeV})\end{array}$ & 0.02 & 0.05 & 0.1 & 0.2 & 0.3 & 0.4 & 0.5 & 0.6 & 0.7 & Spencer \\
\hline \multirow[t]{4}{*}{$\mathrm{Al}$} & 0.16 & - & - & 3.2 & 3.2 & 3.2 & 3.1 & 2.9 & 2.7 & 2.4 & 1.58 \\
\hline & 0.25 & - & - & 3.1 & 3.1 & 3.1 & 3.1 & 2.9 & 2.6 & 2.4 & 1.57 \\
\hline & 0.34 & - & - & 3.1 & 3.1 & 3.1 & 3.1 & 2.9 & 2.7 & 2.5 & 1.56 \\
\hline & 0.96 & - & - & 3.1 & 3.1 & 3.1 & 3.0 & 2.9 & 2.7 & 2.5 & 1.45 \\
\hline \multirow[t]{3}{*}{$\mathrm{Ag}$} & 0.16 & 5.8 & 5.9 & 6.1 & 6.0 & 6.0 & 5.8 & - & - & - & 6.3 \\
\hline & 0.25 & 5.5 & 5.7 & 5.8 & 6.0 & 6.2 & 6.3 & - & - & - & 6.5 \\
\hline & 0.34 & 5.3 & 5.5 & 5.5 & 5.8 & 6.1 & 6.2 & - & - & - & 6.6 \\
\hline \multirow[t]{4}{*}{$\mathrm{Pb}$} & 0.16 & 8.4 & 9.7 & 9.5 & 9.5 & 9.2 & - & - & - & - & 10.0 \\
\hline & 0.25 & 8.2 & 8.4 & 8.7 & 8.7 & 8.9 & - & - & - & - & 10.5 \\
\hline & 0.34 & 7.3 & 7.8 & 8.5 & 8.6 & 8.3 & - & - & - & - & 11.2 \\
\hline & 0.96 & 6.7 & 6.8 & 7.6 & 7.7 & 8.0 & - & - & - & - & 12.3 \\
\hline
\end{tabular}

$K, A$ and $p$ are constants; $R(E)$ is the residual range as defined and tabulated by Nelms ${ }^{19}$ ) of a fast electron, initial energy $E$, in the material.

The value of $K$ follows directly from the boundary condition $x=0, P_{\mathrm{d}}(x, E)=1$ :

$$
K=\exp A \text {. }
$$

Eq. (3) is relatively insensitive to the value of $p . A$ will be sufficiently large that the exponential term dominates. Spencer used integral or half-integral values of $p$ between +1 and -2 . Here the choice

$$
p=-3
$$

is made to simplify the integration.

Eq. (3) has two limitations. Firstly, Spencer used it to extrapolate from a finite to an infinite number of spatial moments; i.e. it applies to the asymptotic tail of $P_{\mathrm{d}}(x, E)$ at deep penetration in the solid and does not necessarily describe the full distribution. Secondly, the Spencer theory does not include range straggling to which the asymptotic tail is particularly sensitive. To investigate these inaccuracies, previous experimental results may be invoked. Seliger ${ }^{17}$ ) has accurately measured $P_{\mathrm{d}}(x, E)$ for a number of materials. He used a $2 \pi$ counter with an efficiency of $99 \%$ down to an electron energy of a few hundred electron volts: an experimental situation entirely analogous to the present work. It is true that the source of electrons was different: a beam impinging perpendicularly on the material, rather than a point isotropic source within the material. However, the electron velocity in the material is quickly randomized, as is evidenced by the back-scattering probability being greater than $50 \%{ }^{20}$ ). Further, Spencer points out that the electron distribution function for a parallel beam source is the same as for a point isotropic source. The two situations are very similar.

The fit of eq. (3) to Seliger's data is demonstrated by the $A$ values in table $1 . A$ is reasonably constant for a given material over the full range of the distribution. The deviation of $A$ from Spencer's theoretical values for the asymptotic tail of the distribution is evident.

The integration of eq. (1) over $x$ may now be made by substituting for $P_{\mathrm{d}}(x, E)$ from eq. (3). With the change of variable

$$
y=\{1-[x / R(E)]\}^{-1}
$$

and the values given by eqs. (4) and (5):

$$
\begin{aligned}
F= & \frac{4}{(h+b)^{2}} \int_{0}^{E_{1}} P_{\mathrm{e}}(E) R(E) \frac{1}{A^{2}} \times \\
& \times[\{2 A R(E)-[h+2 R(E)](A y+1)\} \times \\
& \times \exp \{A(1-y)\}]_{1}^{\infty} \mathrm{d} E .
\end{aligned}
$$

When $y \rightarrow \infty, \exp \{A(1-y)\} \rightarrow 0$. Eq. (6) requires evaluation only for $y=1$, which gives:

$$
\begin{aligned}
F= & \frac{4}{(h+b)^{2} A^{2}} \times \\
& \times \int_{0}^{E_{1}} P_{\mathrm{e}}(E) R(E)[2 R(E)+h(A+1)] \mathrm{d} E ; \\
& R(E)<b / 2 .
\end{aligned}
$$




\subsection{THE ELECTRON ENERGY SPECTRUM}

To complete the evaluation of $F$, a knowledge of the function $P_{\mathrm{e}}(E)$, the probability of a photon interaction producing an electron energy $E$, is required. Two types of photon interaction, the photoelectric effect and Compton scattering, are relevant to the energy range considered here. Pair production starts above $1 \mathrm{MeV}$, but it is still a minor contribution to the total interaction cross section up to $2 \mathrm{MeV}$. The question of photon energies greater than $2 \mathrm{MeV}$ will be the subject of a future paper.

\subsubsection{The photoelectric contribution}

For the case of photon capture by the photoelectric effect, $P_{\mathrm{e}}(E)$ is simply a delta function at the photon energy minus the binding energy of the electron. Two possibilities, $\mathrm{K}$ and $\mathrm{L}+\mathrm{M}$ shells, are treated separately:

$$
\begin{aligned}
& P_{\mathrm{e}}(E)=1, \quad E=E_{\mathrm{ph}}-E_{\mathrm{K}, \mathrm{L}+\mathrm{M}}, \\
& P_{\mathrm{e}}(E)=0, \quad E \neq E_{\mathrm{ph}}-E_{\mathrm{K}, \mathrm{L}+\mathrm{M}} .
\end{aligned}
$$

Putting eqs. (8) into eq. (7):

$$
\begin{aligned}
F_{\mathrm{K}, \mathrm{L}+\mathrm{M}}= & \frac{4 R_{\mathrm{K}, \mathrm{L}+\mathrm{M}}}{(h+b)^{2} A^{2}}\left[2 R_{\mathrm{K}, \mathrm{L}+\mathrm{M}}+h(A+1)\right] ; \\
& R_{\mathrm{K}, \mathrm{L}+\mathrm{M}}<b / 2,
\end{aligned}
$$

where $R_{\mathrm{K}, \mathrm{L}+\mathrm{M}}=R\left(E_{\mathrm{ph}}-E_{\mathrm{K}, \mathrm{L}+\mathrm{M}}\right)$.

\subsubsection{The scattering contribution}

Compton scattering gives rise to a continuous spectrum of electron energy ${ }^{21}$ ). As a reasonable approximation the spectrum may be assumed flat and $P_{\mathrm{e}}(E)$ constant up to the Compton edge energy, $E_{\mathrm{C}}$. Then:

$$
\int_{0}^{E_{\mathrm{C}}} P_{\mathrm{e}}(E) \mathrm{d} E=1 ; \quad \therefore P_{\mathrm{e}}(E)=\frac{1}{E_{\mathrm{C}}} .
$$

For $R(E)$, a range-energy relationship ${ }^{22}$ ) holds:

$$
R(E)=a E^{q},
$$

where the constants $a$ and $a$ follow from Nelms' $\operatorname{data}^{19}$ ).

Substituting eqs. (10) and (11) into eq. (7) and integrating with $E_{1}=E_{\mathrm{C}}$,

$$
\begin{gathered}
F_{\mathrm{C}}=\frac{4 R_{\mathrm{C}}}{(h+b)^{2} A^{2}}\left[\frac{2 R_{\mathrm{C}}}{2 q+1}+\frac{h(A+1)}{q+1}\right] ; \\
R_{\mathrm{C}}<b / 2,
\end{gathered}
$$

where $R_{\mathrm{C}}=R\left(E_{\mathrm{C}}\right)$.
Note that eqs. (9) and (12) are the same for $q=0$ and $R_{\mathrm{C}}=R_{\mathrm{K}, \mathrm{L}+\mathrm{M}}$.

\subsubsection{The escape-peak contribution}

One further small contribution to $F$ should be included. This arises from the de-excitation of an atom following photoelectric capture: the escape peak. Only K-shell de-excitation needs to be considered. An electron resulting from L- or M-shell de-excitation will be of too low an energy to be significant. The de-excitation will either produce an electron immediately (the Auger effect), or after a further photoelectric capture of the photon with the shell energy. The contribution $F_{\mathrm{KE}}$ to detection efficiency by this electron may be treated in the way described in
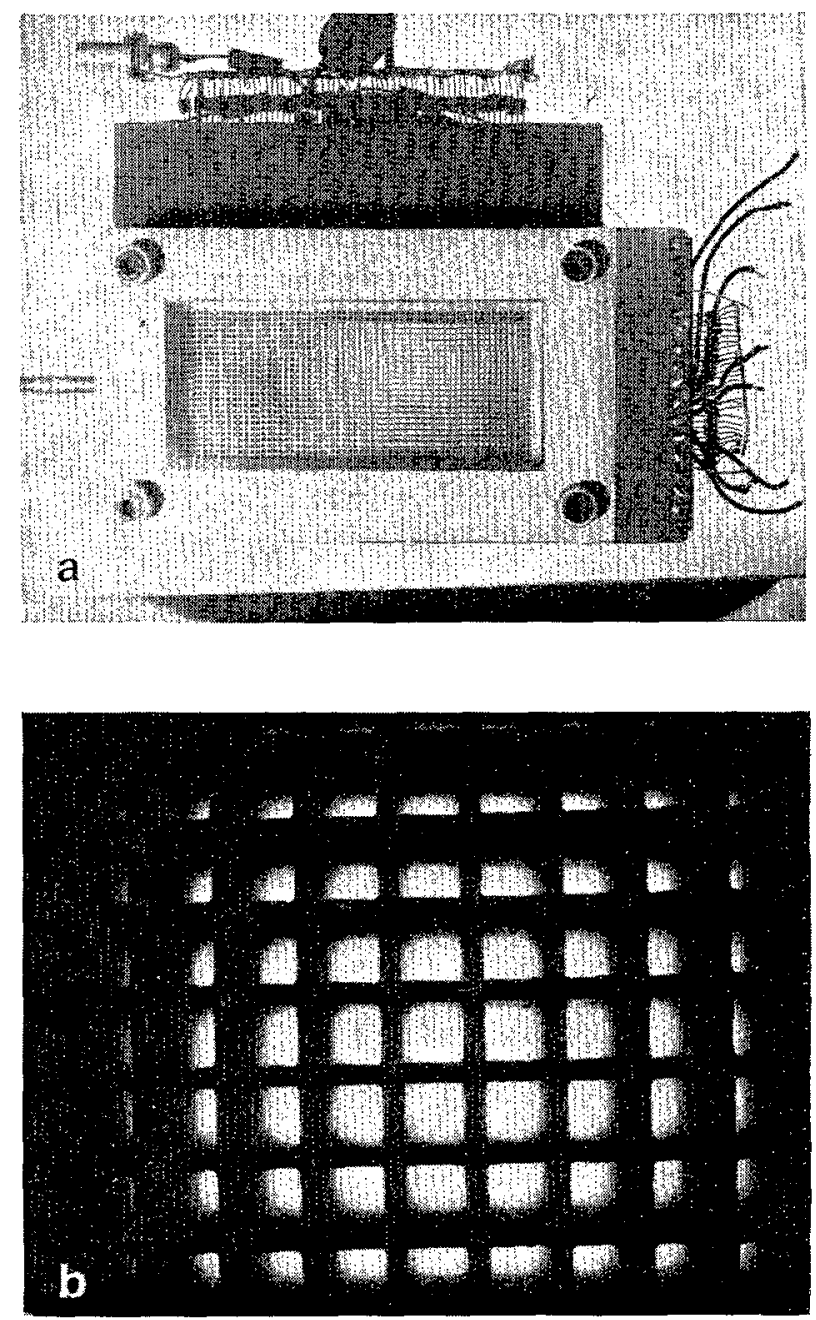

Fig. 5. The lead-bismuth plate chamber: (a) top view $(\times 1 / 3)$ (b) close-up of the plates $(\times 6)$. 
section 3.2.1:

$$
F_{\mathrm{KE}}=\frac{4 R_{\mathrm{KE}}\left[2 R_{\mathrm{KE}}+h(A+1)\right]}{(h+b)^{2} A^{2}},
$$

where $R_{\mathrm{KE}}=R\left(E_{\mathrm{K}}\right), E_{\mathrm{K}}$ being the K-shell energy.

The final stage is to sum the four contributions $F_{\mathrm{K}}$, $F_{\mathrm{L}+\mathrm{M}}, F_{\mathrm{C}}$, and $F_{\mathrm{KE}}$, according to eq. (2). Photon-capture cross sections, and $\mathrm{K}$ to $\mathrm{L}+\mathrm{M}$ shell relative probability have been tabulated by Grodstein ${ }^{23}$ ).

In the next section, the construction and operation of an experimental chamber is described. The results from the theoretical analysis will then be compared with experimental measurements.

\section{An experimental chamber}

\subsection{Chamber CONSTRuCtion}

Fig. 5 shows a small chamber constructed according to the ideas detailed in the previous sections.

To obtain the maximum advantage from the photoelectric effect, lead is a natural choice for the plate material. Since lead is so soft, a eutectic alloy* of lead and bismuth was chosen instead. The matrix was made from 17 plates each cast and machined to a thickness of $0.5 \mathrm{~mm}$. A pattern of square holes, side $1 \mathrm{~mm}$, pitch $1.5 \mathrm{~mm}$, was cut in each plate over an area of $9 \mathrm{~cm} \times 4 \mathrm{~cm}$ by spark-erosion machining. The insulation between the plates was oxidized aluminium foil. Each foil, $0.1 \mathrm{~mm}$ thick, was cut to the same pattern as the lead-bismuth plates by photochemical methods and then anodized to give an oxide layer $0.02 \mathrm{~mm}$ thick on each side. A MPC was positioned $3 \mathrm{~mm}$ beneath the metal stack. Orthogonal cathode planes were of $0.1 \mathrm{~mm}$ diameter, and the anode plane $0.01 \mathrm{~mm}$ diameter, gold-plated tungsten wires. Cathode and anode wire spacings were $1.5 \mathrm{~mm}$, the same as the hole pitch in the plates. The anode wires were centred under the holes, and the cathode wires under the bars, of the matrix. The spacing between wire planes was $3 \mathrm{~mm}$. The chamber was closed with mylar windows.

\subsection{Chamber electronics ANd operation}

The chamber was operated with the cathodes at earth potential and a positive voltage of $2.6 \mathrm{kV}$ on the anode wires. The usual argon-isobutane-methylal gas mixture ${ }^{24}$ ) was used. The resistor chain connected to the plates of the matrix was of $100 \mathrm{k} \Omega$ steps. To obtain a focusing field, the last four resistors were increased in value progressively by $20 \%$. The electric field strength

* Trade name CERRoBASE. Composition $44.5 \% \mathrm{~Pb}, 55.5 \%$ Bi, melting point $124^{\circ} \mathrm{C}$. over the gas space between the matrix and the MPC was double that at the last plate. The drift voltage was $750 \mathrm{~V}$. Exceeding this value caused a big increase in the chamber noise, probably due to the porous nature of the aluminium oxide insulating layers.

Charge-sensitive preamplifiers show considerable advantages for recording pulses from MPCs. A simple three-transistor circuit can give an order of magnitude better signal-to-noise ratio than the usual voltage amplifier with a $10 \mathrm{k} \Omega$ input impedance. Such preamplifiers were made, similar to those described by Radeka ${ }^{25}$ ). They worked well and will be detailed elsewhere.

For pulse counting, the negative anode pulses were used, since they are typically five times larger than the positive cathode pulses. A band of twelve anode wires was connected via an isolating capacitor, to a preamplifier and then to the main amplifier, giving a gain of $60 \mathrm{~dB}$. Some differentiation was applied to remove low-frequency noise. The excellent signal-to-noise ratio of the preamplifier revealed the presence of a secondary pulse about $100 \mu$ s after every main pulse. This was the arrival of the positive argon ions at the high field around the cathode wires. To prevent this pulse, and differentiation overshoots, from counting, a $200 \mu \mathrm{s}$ one-shot was connected between the discriminator and timer-counter (see fig. 6). The consequent counting-rate limitation did not matter, since rates were never greater than a few hundred counts per second.

\section{Chamber performance}

The chamber was investigated to assess its electron drifting action, and its detection efficiency and spatial resolution for photons in the range $0.1-2.0 \mathrm{MeV}$. The experimentally measured efficiencies are compared to the theoretical values derived in section 3 .

\subsection{Electron DRIFTING ACtion}

Testing with an ${ }^{55} \mathrm{Fe}$ source gave results similar to those obtained with the copper matrix, described in section 2: a large increase in count rate, and a flat pulse-

\section{$\square$ Source}

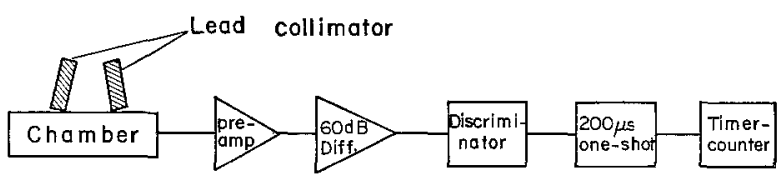

Fig. 6. The electronics arrangement for measuring detection efficiency. 
height spectrum, when a forward-drift voltage was applied. At the maximum chamber voltage, $2.6 \mathrm{kV}$, the $5.9 \mathrm{keV}$ line, which corresponds to two hundred ion pairs in the chamber, gave a pulse height three hundred times the discriminator setting. On the assumption that the chamber was operating in the proportional mode, one ion pair was detectable.

The chamber was next tested with $0.66 \mathrm{MeV}$ photons from a ${ }^{137} \mathrm{Cs}$ source. At this energy, photon conversion by the gas is completely negligible. The source was fixed $10 \mathrm{~cm}$ above the matrix. The photon flux was collimated onto twelve rows of holes by two tilted lead blocks, $5 \mathrm{~cm}$ deep (fig. 6). This prevented spurious counts due to Compton-scattered photons entering the active area from elsewhere in the matrix. Applying the drift voltage produced a sevenfold increase in count rate and a flat pulse-height spectrum. Fig. 7 shows the count rate for a fixed drift voltage as a function of chamber voltage. The saturation at $2.6 \mathrm{kV}$ suggests a detection threshold down to one ion pair, in agreement with the ${ }^{55} \mathrm{Fe}$ observations.

To assess the influence of hole size and plate thickness on the drifting action, the count rate was measured, for different drift voltages, with the plates connected to the resistor chain in singles, doubles, and triples. The count rate saturates in all three cases; very rapidly in the single-plate case (see fig. 8). The saturation values of count rate are plotted against the ratio, hole diameter to plate thickness $(h / t)$, in fig. 9. Now, for

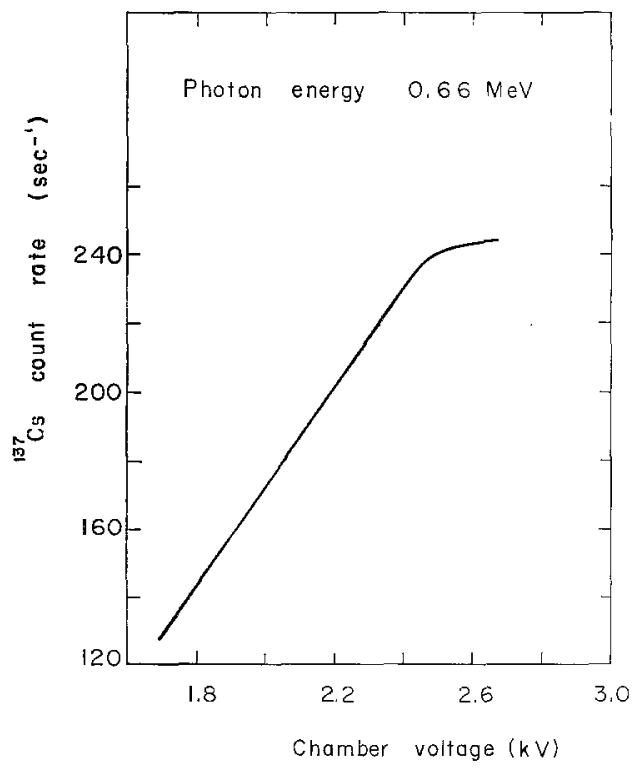

Fig. 7. Count rate as a function of chamber voltage for $0.66 \mathrm{MeV}$ photons. The saturation at $2.6 \mathrm{kV}$ indicates a detection level down to 1 ion pair.

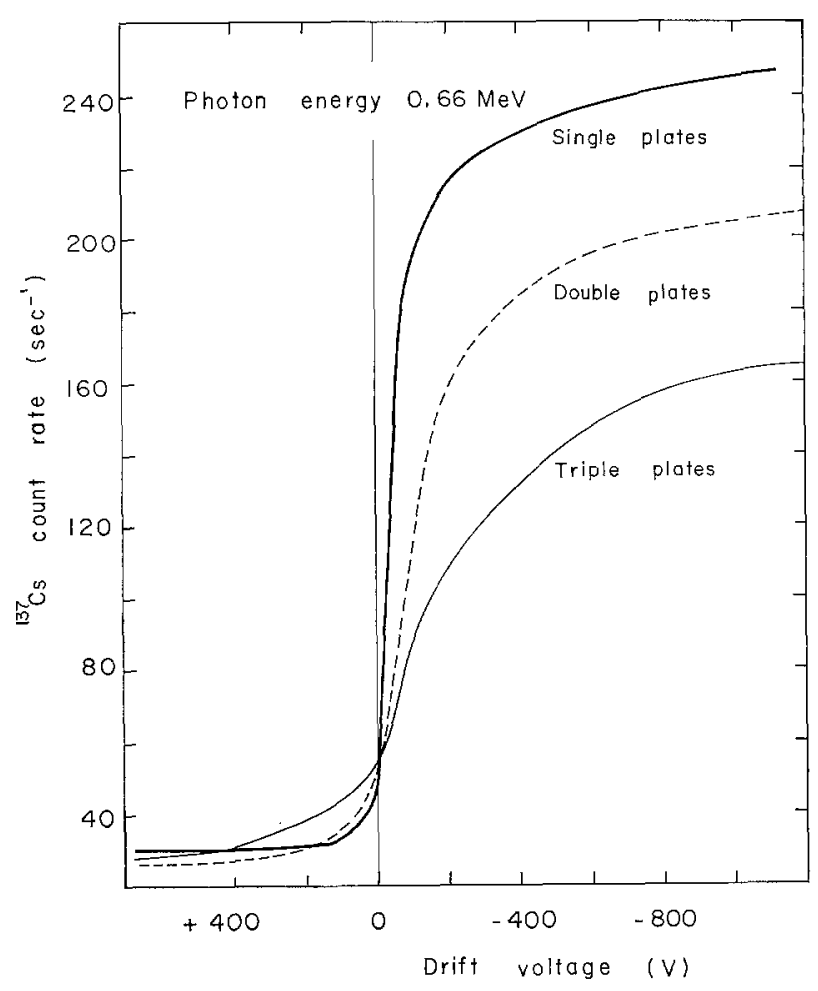

Fig. 8. Count rate as a function of drift voltage for $0.66 \mathrm{MeV}$ photons. The inffuence of plate thickness is shown.

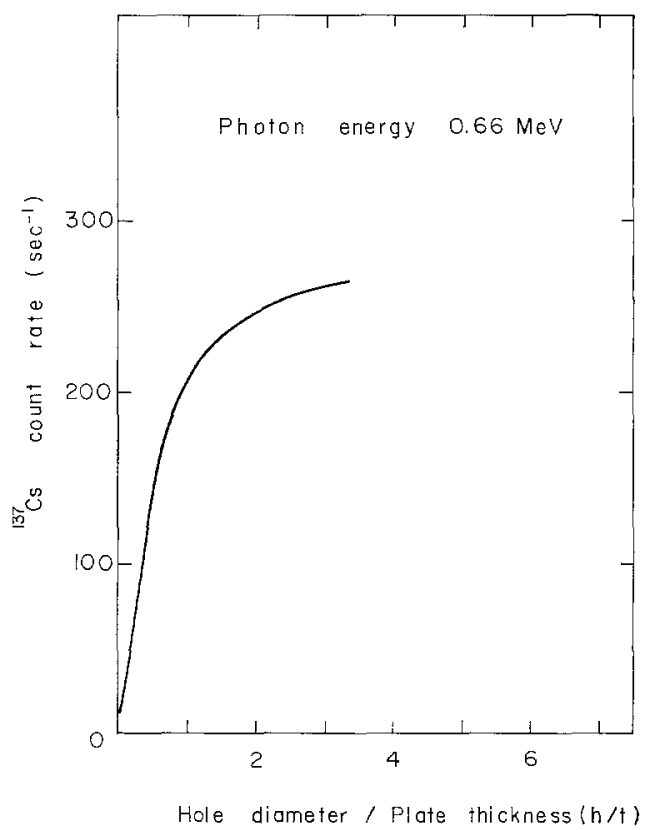

Fig. 9. The influence of plate thickness on the count-rate saturation values of fig. 8 . The saturation here indicates a high probability of electron collection from the matrix. 
TABLE 2

The results of the experimental measurements of detection efficiency.

\begin{tabular}{|c|c|c|c|c|c|}
\hline Source & $\begin{array}{l}\text { Photon } \\
\text { energies } \\
(\mathrm{MeV})\end{array}$ & $\begin{array}{c}\text { Contribution } \\
(\%)\end{array}$ & $\begin{array}{c}\text { MPC } \\
\text { efficiency } \\
(\%)\end{array}$ & $\begin{array}{l}\text { Matrix } \\
\text { efficiency } \\
(\%)\end{array}$ & $\begin{array}{c}\text { Efficiency } \\
\text { ratio }\end{array}$ \\
\hline${ }^{57} \mathrm{Co}$ & $\begin{array}{l}0.014 \\
0.122 \\
0.136 \\
0.707\end{array}$ & $\left.\begin{array}{c}7.8 \\
84.8 \\
11.4 \\
0.2\end{array}\right\}$ & $\begin{array}{l}0 \\
-\end{array}$ & $\begin{array}{r}2.2 \\
-\end{array}$ & 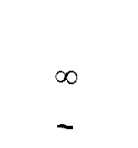 \\
\hline${ }^{203} \mathrm{Hg}$ & $\begin{array}{c}0.071 / 0.085 \\
0.279\end{array}$ & $\begin{array}{l}12.7 \\
81.5\end{array}$ & 0.24 & 3.33 & 13.9 \\
\hline${ }^{22} \mathrm{Na}$ & $\begin{array}{l}0.511 \\
1.275\end{array}$ & $\begin{array}{l}181 \\
100\end{array}$ & 0.58 & 3.46 & 6.0 \\
\hline${ }^{137} \mathrm{Cs}$ & $\begin{array}{c}0.032 / 0.037 \\
0.662\end{array}$ & $\begin{array}{r}8.1 \\
85.1\end{array}$ & 0.61 & 4.37 & 7.2 \\
\hline${ }^{54} \mathrm{Mn}$ & 0.835 & 100 & 0.73 & 5.16 & 7.1 \\
\hline${ }^{60} \mathrm{Co}$ & $\begin{array}{l}1.173 \\
1.332\end{array}$ & $\left.\begin{array}{l}100 \\
100\end{array}\right\}$ & 1.03 & 7.0 & 6.8 \\
\hline${ }^{88} \mathrm{Y}$ & $\begin{array}{l}0.898 \\
1.836 \\
2.76\end{array}$ & $\begin{array}{r}91.5 \\
99.5 \\
0.5\end{array}$ & $\begin{array}{r}1.7 \\
-\end{array}$ & $\begin{array}{c}11.3 \\
-\end{array}$ & $\begin{array}{l}6.7 \\
-\end{array}$ \\
\hline
\end{tabular}

large values of $h / t$, the chamber will look like a gas drift space $(h / t \rightarrow \infty)$, which is well known to be capable of drifting electrons with no losses. From the saturation of fig. 9 one sees, therefore, a clear indication of efficient drifting action by the matrix, provided $h / t>2$. Some changes were made to the resistor chain for the single-plate connection. No significant change from fig. 8 was observed. However, it is possible that the situation $h / t<2$ could be improved by a careful optimization of resistor values.

A far more detailed experimental investigation would clearly be of interest; for example to determine the pulse-height spectrum as a function of drift voltage, resistor values, and photon energy, and to look at much thicker stacks. In general, it may be said that for a $1 \mathrm{~cm}$ stack the drifting action for count rate is very efficient, provided the hole diameter is at least twice the plate thickness.

\subsection{The DETECTION EFFICIENCY}

The detection efficiency was measured as a function of photon energy by replacing the ${ }^{137} \mathrm{Cs}$ source used previously with each of a number of calibrated sources*, details of which appear in table 2 .

* Gamma Reference Source Set, The Radiochemical Centre, Amersham, England.

\subsubsection{Measurement procedure}

Three measurements of count rate were made at each energy for both forward- and backward-drift fields. Subtraction separates the direct chamber and matrix contributions. "No source" background, being the sum of cosmic and natural radiation and chamber discharges, was also measured and subtracted. For the ${ }^{22} \mathrm{Na}$ source, thin aluminium discs were placed in contact with the source to ensure complete positron annihilation close to it. A $2 \mathrm{~mm}$ thick sheet of lucite was interposed between the chamber and the ${ }^{137} \mathrm{Cs}$ source to absorb the $0.63 \mathrm{MeV}$ electron radiation.

Only the ${ }^{54} \mathrm{Mn}$ source provides monoenergetic photons. But the ${ }^{60} \mathrm{Co}$ lines are sufficiently close in energy that the source may be considered as giving monoenergetic photons at the average energy. Conveniently, the results from these two sources may be used to resolve the double lines of ${ }^{88} \mathrm{Y}$ and ${ }^{22} \mathrm{Na}$. For ${ }^{57} \mathrm{Co}$, the $0.707 \mathrm{MeV}$ line may be ignored; a calculated gas capture correction applied for the $0.014 \mathrm{MeV}$ photons and the other two energies averaged. The weak, low-energy lines of ${ }^{203} \mathrm{Hg}$ and ${ }^{137} \mathrm{Cs}$ were allowed for by extrapolating the ${ }^{57} \mathrm{Co}$ result, together with gas capture corrections.

\subsubsection{Experimental results}

Results are given in table 2 . The detection efficiency 
due to the MPC varies from $0.25 \%$ at $0.28 \mathrm{MeV}$ to $1.7 \%$ at $1.8 \mathrm{MeV}$. A comparison may be made with the known efficiency of a cylindrical Geiger counter over this energy range ${ }^{13}$ ) (fig. 10). A consistent picture is seen. At high energies the MPC looks like a leadwalled Geiger counter and at low energies like an aluminium-walled counter. This is explained by the screening effect of the matrix at low energies. The attenuation length for $0.1 \mathrm{MeV}$ photons in lead is $0.2 \mathrm{~mm}$. Only those photons that pass through the holes in the matrix and convert in the MPC, presumably on the bottom mylar window, may be recorded. At higher energies the photons penetrate the matrix and the MPC receives Compton electrons and photoelectrons from the bottom lead-bismuth plate.

The matrix efficiency is $1.8 \%$ at $0.1 \mathrm{MeV}$, rising to $11 \%$ at $1.8 \mathrm{MeV}$. It should be noted that the total photon interaction probability for the matrix is $18 \%$ at $1.8 \mathrm{MeV}$. Thus $60 \%$ of the photons that interact are detected. The ratio matrix efficiency to MPC efficiency is roughly constant, above $0.5 \mathrm{MeV}$, at 7 , a value measured previously (section 5.1). At the lower energies this ratio increases, reflecting the rapidly decreasing MPC efficiency, explained above.

In fig. 11 the theoretical calculation of the matrix detection efficiency, described in section 3, is compared. with the experimental results. The four contributions: K-shell $\left(\varepsilon_{\mathrm{K}}\right)$ and $\mathrm{L}, \mathrm{M}$-shells $\left(\varepsilon_{\mathrm{L}}\right)$ photoelectric capture, Compton scattering $\left(\varepsilon_{\mathrm{C}}\right)$, and the $\mathrm{K}$-shell escape peak $\left(\varepsilon_{\mathrm{KE}}\right)$ are shown, together with the total $\left(\varepsilon_{\mathrm{T}}\right)$. The general theoretical-experimental agreement is very pleasing. Two small discrepancies are evident: the experimental

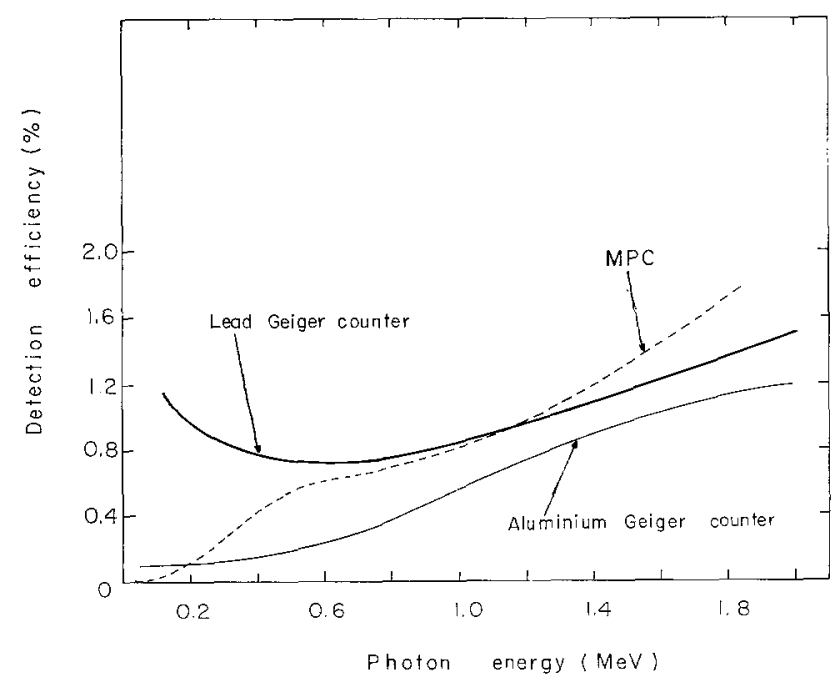

Fig. 10. A comparison of the MPC efficiency with the known efficiencies of lead and aluminium Geiger counters. values exceed the theoretical above $1.0 \mathrm{MeV}$ and below $0.4 \mathrm{MeV}$. There are two explanations for the high-energy discrepancy. Firstly, the basic condition $R(E)<b / 2$ (see section 3 ) of the theoretical model is violated above $0.4 \mathrm{MeV}$. In fact, one sees from Seliger's data that this condition is too severe. For lead, the electron escape probability is effectively zero at $\frac{1}{2} R(E)$. Thus the condition relaxes to $R(E)<b$, which is not violated until $0.8 \mathrm{MeV}$, just where the discrepancy begins to appear. The second factor is the onset of pair production above $1.0 \mathrm{MeV}$. At $1.8 \mathrm{MeV}$ the pairproduction cross section is $6 \%$ of the total. However, the electron-positron pair will average only $0.4 \mathrm{MeV}$ each in kinetic energy: $1.0 \mathrm{MeV}$ of energy is lost in creating the pair. And the subsequent annihilation of the positron will not contribute significantly to the efficiency: detecting the resulting $0.5 \mathrm{MeV}$ photons is a second-order process. One would expect only a fraction of a per cent extra in efficiency.

The reason for the low-energy discrepancy is less obvious. It is probably due to an extra contribution from the upper surface of the top plate of the matrix. As pointed out above, the attenuation length of $0.1 \mathrm{MeV}$ photons in lead is $0.2 \mathrm{~mm}$. Nearly all the photon capture will be in the first plate, at this energy; and fig. 10 shows a sharp increase in the efficiency of a lead Geiger counter below $0.4 \mathrm{MeV}$.

\subsection{Spatial resolution}

The centre-of-gravity method $^{10}$ ) was applied to single cathode wires to measure the spatial resolution. In fig. 12 are displayed histograms of the number of

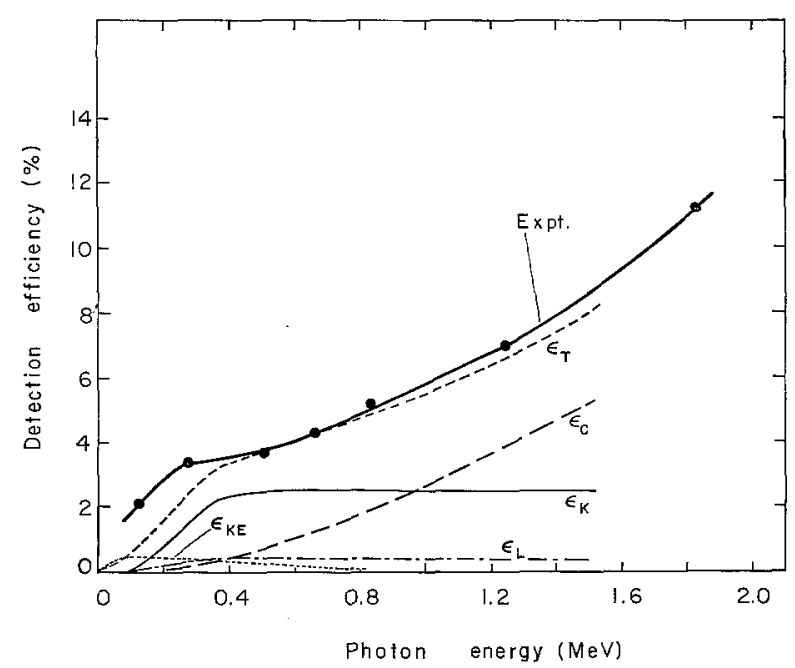

Fig. 11. A comparison of the experimental and theoretical efficiencies of the matrix. $\varepsilon_{\mathbf{T}}=\varepsilon_{\mathrm{KE}}+\varepsilon_{\mathrm{L}}+\varepsilon_{\mathrm{K}}+\varepsilon_{\mathrm{C}}$. 
events as a function of position along the anode wires, i.e. the direction of continuous resolution. Each tick mark represents $0.5 \mathrm{~mm}$. Fig. 12a shows the result for an uncollimated $0.66 \mathrm{MeV}{ }^{137} \mathrm{Cs}$ source, with a backdrift voltage, i.e. the MPC background. When the forward-drift voltage is applied, the hole structure of the matrix is revealed (fig. 12b). The peaks are narrower than the hole size because of the focusing action of the drift field. The source was collimated with a $0.5 \mathrm{~mm}$ wide slot in a lead block $5 \mathrm{~cm}$ deep. The slot was positioned along a row of holes perpendicular to the anode wires. Fig. $12 \mathrm{c}$ shows, as expected, that the spatial resolution is basically the hole size. The envelope of the three peaks has a fwhm of $1.3 \mathrm{~mm}$. Finally, in fig. $12 \mathrm{~d}$, one sees the result from a collimated ${ }^{60} \mathrm{Co}$ source $(1.25 \mathrm{MeV})$. The resolution is lost because of the dominance of Compton scattering over the photoelectric effect at this energy. At $0.66 \mathrm{MeV}$ the photoelectric-to-Compton cross-section ratio is about $1: 1.5$, and at $1.25 \mathrm{MeV}, 1: 3$. This is an important result: it
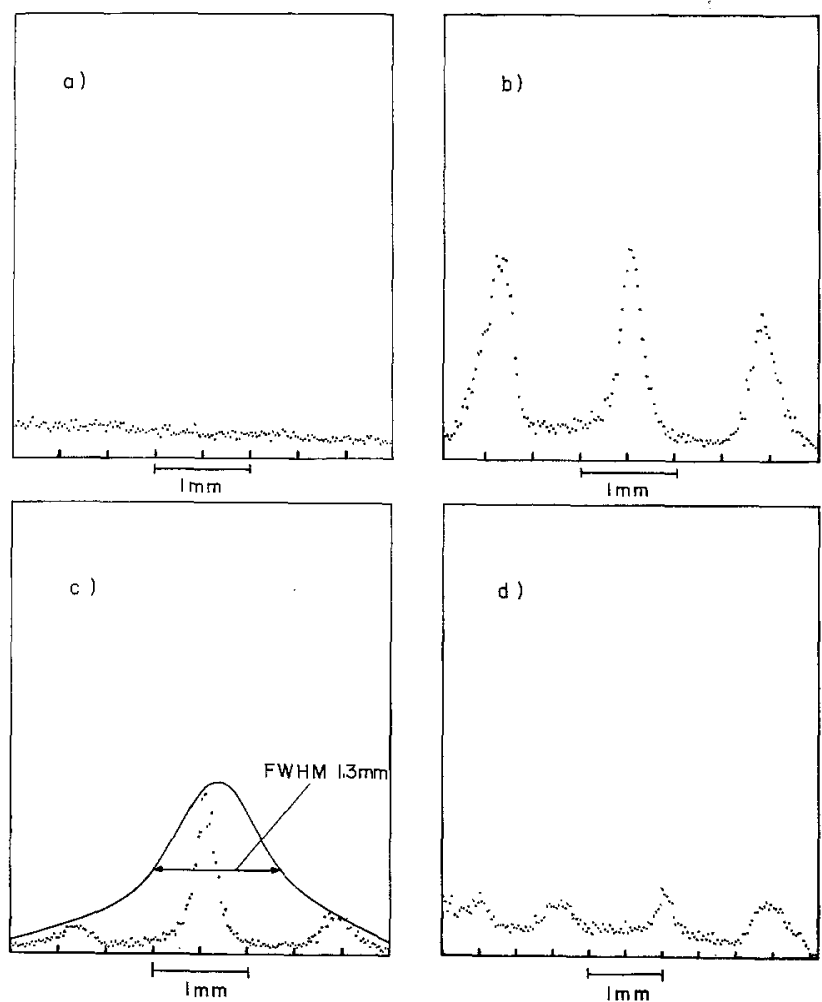

Fig. 12. Spatial resolution given by the matrix. $0.66 \mathrm{MeV}$ photon source: (a) Uncollimated source, back-drift voltage; (b) Uncollimated source, forward-drift voltage; (c) Collimated source, forward-drift voltage. $1.25 \mathrm{MeV}$ photon source: (d) Collimated source, forward-drift voltage. Spatial resolution is lost at high photon energies due to the predominance of Compton scattering. clearly demonstrates the need for photoelectric capture to obtain a good spatial resolution.

Figs. 13a-e show the sequence obtained by moving the collimated $0.66 \mathrm{MeV}$ source across the matrix in half-pitch $(0.75 \mathrm{~mm})$ steps. The passage of the beam from hole to hole may be clearly followed. Indeed a source movement of $0.05 \mathrm{~mm}$ caused an observable change in the relative heights of the peaks.

\section{Conclusion}

The gas-solid hybrid MPC described here offers new possibilities for the spatial localization of nonionizing radiation, over a very wide energy range.

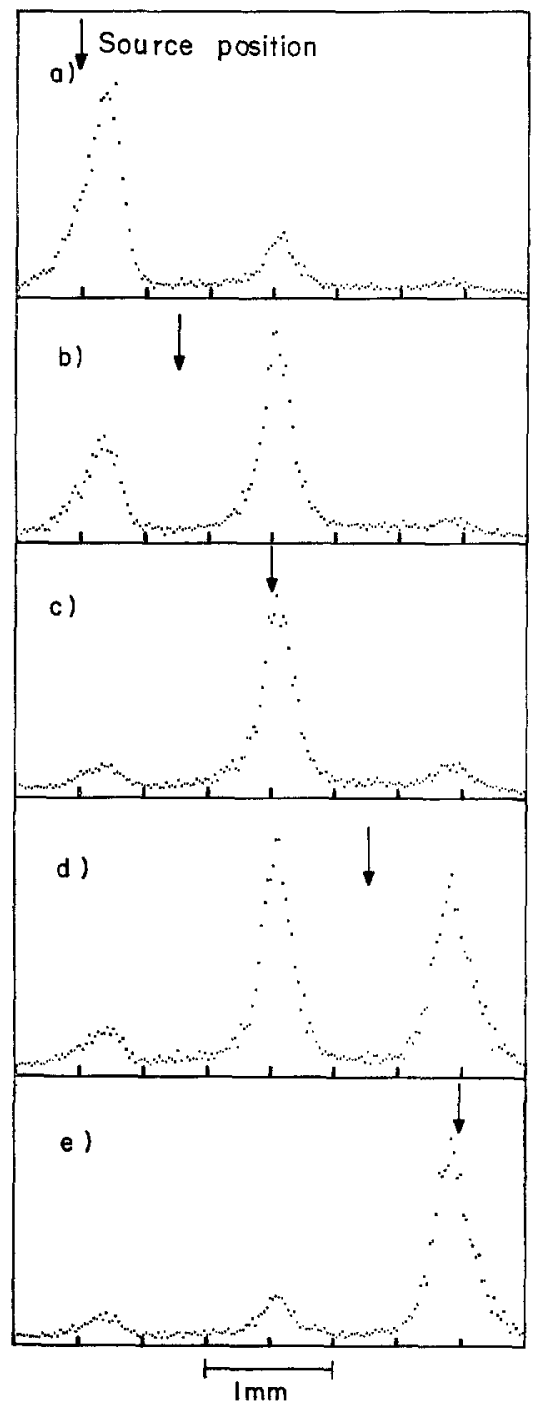

Fig. 13. (a)-(e) The passage of a collimated beam of $0.66 \mathrm{MeV}$ photons from hole to hole of the matrix, in steps of $0.75 \mathrm{~mm}$. 
These possibilities stem from two basic properties of the solid matrix:

i) Good detection efficiency due to the high probability of interaction of the non-ionizing radiation with the solid.

ii) Good spatial resolution due to the hole size limitation to the range of the ionizing products.

Advantage may be taken of either or both of these properties, depending on the nature and energy of the radiation.

It has been shown that both properties are fundamental for detecting and localizing photons in the energy range $0.1-2.0 \mathrm{MeV}$. At $1.8 \mathrm{MeV}, 60 \%$ of the photons interacting with the solid are detected. Spatial resolution of $1.3 \mathrm{~mm}$ fwhm is obtained below $1.0 \mathrm{MeV}$, where the photoelectric effect is dominant. Energy resolution is lost, however. By correct choice of matrix material and geometry and by using multiple stacks, such results should be possible for any particular energy within the $0.1-2.0 \mathrm{MeV}$ range. Of particular interest is $0.5 \mathrm{MeV}$ for positron work and around $0.1 \mathrm{MeV}$ for general radio-isotope imaging. If the matrix is made of neutron-sensitive material, for example boron, lithium, or gadolinium, similar results should be forthcoming for thermal and epithermal neutrons. An extension to fast-neutron detection should be possible by replacing the matrix insulation with sheets of hydrogeneous material.

Using the property of range limitation alone is applicable to the case where the gas provides good detection efficiency, but insufficient spatial resolution. An obvious example is the use of ${ }^{3} \mathrm{He}$ for thermalneutron imaging ${ }^{26}$ ). At the expense of energy resolution, the use of a matrix would remove the need for pressurization or addition of a stopping gas, such as krypton. The same argument applies to using argon for detecting $20-50 \mathrm{keV}$ photons, or xenon for $50-100 \mathrm{keV}$. Whether the matrix could be made in a spherical form, for use in a spherical drift chamber, is perhaps an intriguing technological problem.

At very much higher energies $(\mathrm{GeV})$ the range limitation property disappears, but the high detection efficiency remains. The gas-solid matrix becomes a high-density drift space; using dense metals such as tungsten, more than $10 \mathrm{~g} / \mathrm{cm}^{3}$ should be possible. One then has a shower detector that should compete very favourably with the liquid-argon ${ }^{27}$ ) or lead-glass ${ }^{28}$ ) detectors. By making use of drift-time as well as spatial information, three-dimensional siting of the shower vertex should be obtained. Further, if the sampling of the shower from the gas ionization is adequate, energy resolution will be regained.
A general program of work is under way to explore the varied and very promising topics outlined above.

We are greatly indebted to Messrs. K. Kull, G. Dinkel and J. Toustou for constructing the chamber, and Messrs. C. Parkman, J. C. Santiard and Z. Hajduk for assisting with the electronics.

We thank Prof. M. Peter, and Drs M. G. N. Hine, G. R. Macleod, P. Zanella and H. Davies for their enthusiastic interest and support.

\section{References}

1) G. Charpak, Ann. Rev. Nucl. Sci. 20 (1970) 195.

2) A. H. Walenta, J. Heintze and B. Schürlein, Nucl. Instr. and Meth. 92 (1971) 373.

3) J. Saudinos, J.-C. Duchazeaubeneix, C. Laspalles and R. Chaminade, Nucl. Instr. and Meth. 111 (1973) 77.

4) S. N. Kaplan, L. Kaufman, V. Perez-Mendez and K. Valentine, Nucl. Instr. and Meth. 106 (1973) 397.

5) C. Cork, D. Fehr, R. Hamlin, W. Vernon, N. H. Xuong and V. Perez-Mendez, private communication.

6) K. Valentine, S. N. Kaplan, V. Perez-Mendez and L. Kaufman, IEEE Trans. Nucl. Sci. NS-21 (1974) 178.

7) C. J. Borkowski and M. K. Kopp, IEEE Trans. Nucl. Sci. NS-17 (1970) 340.

8) V. Perez-Mendez and S. I. Parker, IEEE Trans. Nucl. Sci. NS-21 (1974) 45.

9) W. R. Kuhlmann, K. H. Lauterjung, B. Schimmer and K. Sistemich, Nucl. Instr. and Meth. 40 (1966) 118.

10) G. Charpak, A. P. Jeavons, F. Sauli and R. J. Stubbs, CERN 73-11 (1973).

11) G. Charpak, Z. Hajduk, A. P. Jeavons, R. Kahn and R. J. Stubbs, Nucl. Instr. and Meth. 122 (1974) 307.

12) S. E. Derenzo, T. F. Budinger, R. G. Smits, H. Zaklad and L. W. Alvarez, Proc. Symp, on Advanced technology arising from particle physics research, Argonne, 1973 (ANL 8080, Argonne, Ill., 1973), p. 11, 1.

13) S. C. Curran and J. D. Craggs, Counting tubes (Butterworth, London, 1949), p. 89.

14) C. B. Lim, D. Chu, L. Kaufman, V. Perez-Mendez and J. Sperinde, IEEE Trans. Nucl. Sci. NS-21 (1974) 85.

15) L. V. Spencer, Phys. Rev. 98 (1955) 1597.

16) L. V. Spencer, Energy dissipation by fast electrons, N.B.S. Monograph (Nat. Bureau of Standards, Washington, 1959).

17) H. H. Seliger, Phys. Rev. 100 (1955) 1029.

18) E. Segrè, Experimental nuclear physics (J. Wiley, New York, 1953), Vol 1, p. 292.

19) A. T. Nelms, Energy loss and range of electrons and positrons (Nat. Bureau of Standards, Washington; circ. 577, 1956).

20) H. H. Seliger, Phys. Rev. 88 (1952) 408.

21) R. D. Evans, in Handbuch der Physik (Springer Verlag, Berlin, 1958), Vol. 34, p. 268.

22) R. D. Birkhoff, in Handbuch der Physik (Springer Verlag, Berlin, 1958), Vol. 34, p. 132.

23) G. W. Grodstein, X-ray attenuation coefficients from $10 \mathrm{keV}$ to $100 \mathrm{MeV}$ (Nat. Bureau of Standards, Washington; circ. $583,1957)$.

24) G. Charpak, H. G. Fisher, C. R. Gruhn, A. Minten, F. Sauli 
and G. Plch, Nucl. Instr. and Meth. 99 (1972) 279.

25) V. Radeka, IEEE Trans. Nucl. Sci. NS-21 (1974) 51.

26) B. H. Meardon and D. C. Salter, Rutherford lab. rep. RHEL/R 262 (1972), p. 12.

27) S. E. Derenzo, A. Schwemin, R. G. Smits, H. Zaklad and L. W. Alvarez, Berkeley report LBL-1791 (1973).
28) J. Beale, F. W. Büsser, L. Camilleri, L. Di Lella, G. Gladding, A. Placci, B. G. Pope, A. M. Smith, B. Smith, J. K. Yoh and E. Zavattini; B. J. Blumenfeld and L. M. Lederman; R. L. Cool, L. Litt and S. L. Segler, Proc. Intern. Conf, on Instrumentation for high-energy physics, Frascati, 1973 (Lab. Naz. CNEN, Frascati, 1973), p. 415. 